A $R$ R T T I I C C U L L O O $\begin{array}{lllllllll}R & E & F & L & E & X & I & O & N\end{array}$

\title{
DifUSIÓN DE DRAMAS COREANOS, UN ANÁLISIS DE SU ÉXODO A AMÉRICA Latina y Colombia
}

DIFFUSION OF KOREAN DRAMAS, AN ANALYSIS OF ITS EXODUS TO LATIN AMERICA AND COLOMBIA

Por: Liz Andrea Zarco*

Recibido: 18 de febrero de 2018 - Aprobado: 30 de mayo de 2018

* Comunicadora Social de la Universidad de Cartagena. Miembro del semillero de investigación "Comunicación Educación y Cultura" adscrito a la Facultad de Ciencias Sociales y Educación de la misma institución.
1 De ahora en adelante, Corea se utilizará para hacer referencia a la República de Corea comúnmente conocida como Corea del Sur. 2 Este concepto se utiliza en su idioma original. De ahora en adelante se empleará como sinónimo de la ola coreana.

\section{RESUMEN}

En el presente ensayo se pretende realizar un análisis de la difusión de los dramas coreanos en América Latina y Colombia, partiendo de una breve descripción del movimiento Hallyu, su origen en Asía. Aunque se establece que las relaciones diplomáticas, las relaciones comerciales y los procesos migratorios fueron factores determinantes que hacen posible su llegada al continente, se plantea la hipótesis de que estos no aplican para el caso de Colombia.

Palabras clave: América Latina, Hallyu, Ola coreana.

\begin{abstract}
In the present essay, an analysis of the diffusion of Korean dramas in Latin America and Colombia is attempted, starting from a brief description of the Hallyu movement, its origin in Asia. Although it is established that diplomatic relations, trade relations and migratory processes were determining factors that make it possible to reach the continent, the hypothesis is that these do not apply to the case of Colombia.
\end{abstract}

Keywords:Latin America, Hallyu, Korean Wave.

\section{Introducción}

$\mathrm{E}$ ntender que un producto audiovisual producido en el sudeste asiático sea transmitido en los televisores, en lugares distantes geográfica y culturalmente donde las barreras idiomáticas son evidentes, y que además esté disponible en streaming en portales especializados, es producto de los vaivenes de la globalización. El caso que nos concierne, el de Corea ${ }^{1}$, es un claro ejemplo de lo anterior.

La denominada 'Ola coreana' o Hallyu ${ }^{2}$ es definida por numerosos autores (López, 2012; Doobo, 2006; Kim, 2007; Ryoo, 2009; Lee, 2011, entre otros) 
coincidiendo en que se refiere a la consolidación de la estrategia de expansión, a partir de fondos privados y estatales, de todo tipo de productos propios de la cultura popular coreana en los que se incluyen principalmente dramas televisivos, películas, canciones pop, celebridades o idols, comida, entre otros aspectos inicialmente hacia mercados asiáticos con una posterior repercusión global. Hallyu hoy en día es sinónimo de una sólida distribución, consumo y de altos índices de recepción favorable de la cultura popular coreana en Asia, y en el resto del mundo.

¿Cómo llegamos hasta aquí? La industria de entretenimiento coreana tomó como punto de partida para la internacionalización de sus productos culturales a la sociedad china, ¿por lo que para 1997 ya estaría al aire en la Televisión Central de China (CCTV) un drama titulado "What is love all about?" o ¿Qué es el amor?, batiendo records en audiencia en Pekín. (López, 2012:585). Seguidamente, el éxito se repetiría con el drama Jealous de MBC-TV que fue importado como el primer producto cultural coreano en 1993 (Kim, 2007:15 citado en Lee, 2011:86) Dos años más tarde, en respuesta a la demanda esta vez con drama Stars in my heart o "Un deseo de las estrellas" cautivaría al público chino y taiwanés. (López, 2012:585-586) Sin embargo, se tiene certeza de que el fenómeno comenzó propiamente al final de la década de los 90, cuando en diciembre del $2000 \mathrm{el}$ periódico The Peoples Daily utilizó este término para describir el gran recibimiento de los dramas o telenovelas coreanas por parte de la audiencia china. (Zhang, 2006 citado en López, 2012).

Este hecho representaría para las industrias culturales asiáticas un gran impacto puesto que Corea, siendo un país históricamente marginado por parte de las potencias vecinas, dividido a causa de la guerra y al mismo tiempo se encontrase en un crecimiento exponencial económico, fuese el promotor de una nueva forma de

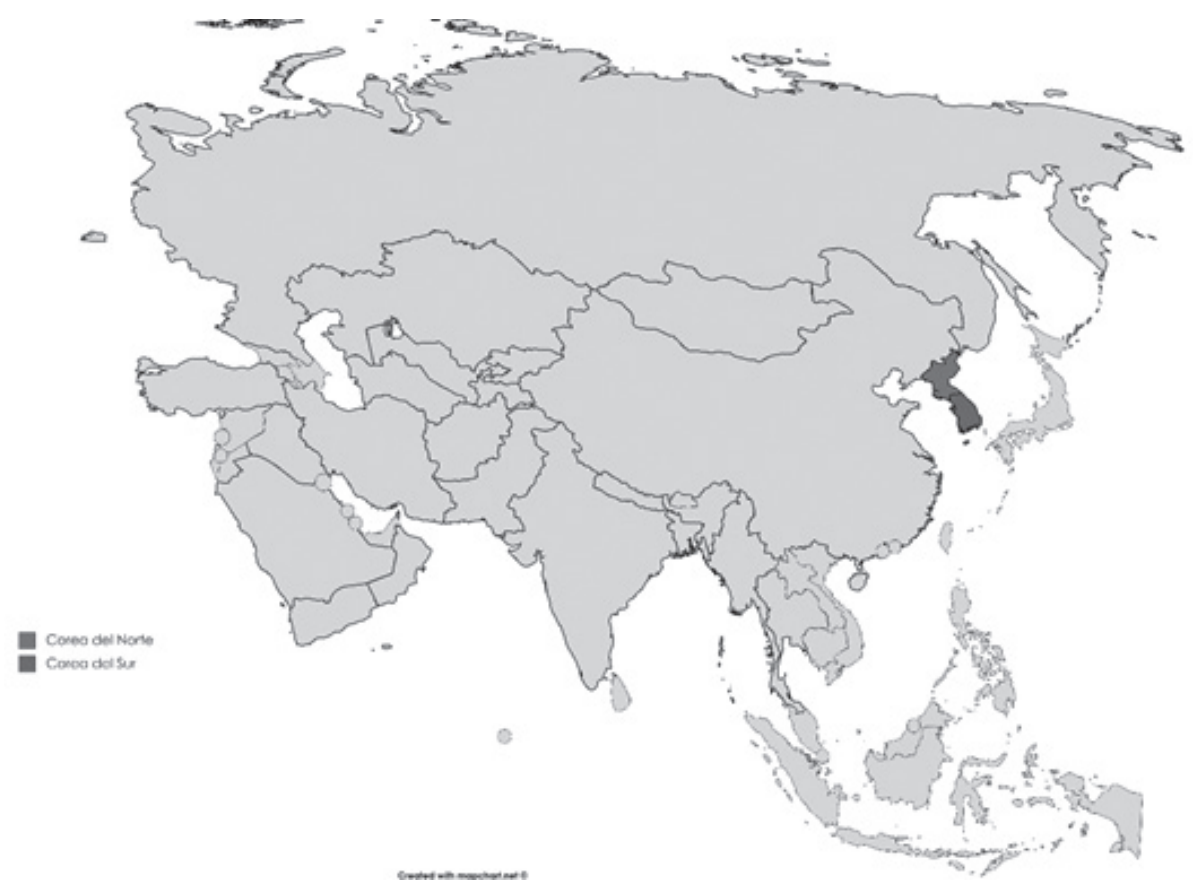

Mapa 1: las dos Coreas

Fuente: Elaboración propia 
3 Película coreana cuya trama se desarrolla a partir de la representación de las prácticas musicales tradicionales de este país.

4 Modelo económico coreano que surge a partir del apoyo político y financiero privilegiado por parte del Estado a grandes conglomerados o empresas privadas de control familiar, para el crecimiento económico después de la independencia de ese país. Un ejemplo son las empresas Samsung, Hyundai, Daewoo, entre otros. (Bustelo, 1991) unión regional por su cultura popular. En este sentido, Corea entraría a competir en un mercado donde la cultura pop estaría dominada por Tokio y Hollywood (Visser, 2003 citado en Doobo, 2006: 22).

Para Doobo (2006) la liberalización de los medios en Asia desde finales de los años 80 y mitad de los 90, fue el principal factor para la difusión de los productos televisivos coreanos, que alcanzaron gran popularidad en países como Hong Kong, Taiwán, Singapur, Vietnam e Indonesia, también liberalizados a comienzos de 1990. Por lo tanto, los productos coreanos representarían competencia al ofrecer su programación a un cuarto del precio japonés y una décima parte de los originarios de Hong Kong a partir del 2000. (Lee, 2003 citado en Doobo, 2006). No obstante, después del auge en la demanda de estos productos, para 2005 el precio promedio de los dramas coreanos sería mucho más costoso que los hollywoodenses o japoneses (Kim, 2007: 123).

Antes de la liberalización de los medios, el panorama para la industria cultural en Corea no era nada alentador. En 1988, mismo año en el que Seúl sería sede de los Juegos Olímpicos de Verano, el permiso de distribución de las películas extranjeras pasaría de las compañías nacionales a manos de Hollywood debido a presión de los Estados Unidos. Aquello supondría un serio problema para el desarrollo de la industria cinematográfica nacional y cultural, el cual era ignorado por la audiencia coreana, junto al crecimiento en la programación extranjera en televisión.

Es por esto que, en 1994, después del éxito nacional de la película Sopyonje ${ }^{3}$ (1993), el gobierno apoyó la industria cultural local a través de la creación de la Oficina de la Industria Cultural dentro del Ministerio de Cultura y Deporte, y de la ley de promoción cinematográfica para atraer el capital corporativo e inversión. (Doobo, 2006: 31-32) Esa acción gubernamental demuestra el apoyo igualitario para la naciente industria cultural en comparación con la dada a las empresas dedicadas a la fabricación de aparatos electrónicos y automóviles (Kim, 2007: 124).

Si bien la industria cultural coreana estuvo hasta ese entonces permeada por Hollywood, debía aprender de ella para crear una propia. Es por esto que, años más tarde el rol que representarían los $c^{2} a e{ }^{4}{ }^{4}$, sería esencial para expansión de este sector en la producción, importación, distribución y exhibición; y, cuya participación duraría hasta la crisis de 1997 y continuaría 
la orientación hacia las exportaciones en la economía de ese país. Como consecuencia, ese corto boom caracterizado por la promoción por parte del gobierno y la inversión de capital privado, ayudó a fortalecer la industria cinematográfica coreana y la posterior inversión de firmas inversoras. (Doobo, 2006: 32-33) En efecto, la importación de programas de televisión extranjeros decaería debido al incremento en la oferta local, los efectos de la mencionada crisis asiática y al aumento en los roles de las productoras independientes (Jin, 2004, en Kim, 2007:122), formando un escenario ideal para la consolidación interna de los productos audiovisuales propios de la cultura popular coreana.

Paralelamente, la industria musical también estaría en auge. Para los años 80 , el mercado estaría dominado por las baladas y los ppongjjak ${ }^{5}$ a pesar del panorama orientado a la preferencia de canciones americanas por parte de los jóvenes coreanos, y el control de la escena musical por dos cadenas de televisión pública, Korea Broadcasting System (KBS) y Munhwa Broadcasting Company (MBC), especialmente en la distribución de la música y la dirección su consumo, influenciando a los grupos musicales por sus estándares para participación. En 1992, aparecería Seo Taiji and Boys, la primera banda en convertirse en pop stars por los siguientes aspectos innovadores: 1) por su popularidad basada en la hibridación innovadora de la música con la mezcla de diferentes géneros e incluso ppongjjak; 2) por la expansión de lo que se conocería como $K-p o p^{6}$ en el mercado musical; 3) por el repertorio con contenido político y social; 4) por desafiar a las cadenas de televisión que dominaban el mercado, abriendo paso a los futuros boy bands y expandiendo los roles de las compañías discográficas y agencias de talento.

Por lo tanto, la hibridación de las formas musicales y la industrialización de creación de pop stars en el K-pop, cumplen un papel importante en la preparación de la cultura popular coreana para la incursión en los mercados regionales; siendo además un signo de resistencia del subalterno, considerando la amplia dominación americana en las industrias culturales globales (Doobo, 2006: 35-40).

La recepción de los productos culturales coreanos en sus países vecinos se explica a partir de la proximidad cultural y geográfica existente. Aunque tuvieron una reacción común en la aceptación, cada país lo recibió desde una perspectiva ligeramente diferente (Lee, 2011: 86) En ese sentido, Ryoo (2009) afirma que:

5 Estilo de música influenciada por Japón que fue asimilada por Corea en el siglo XX (Shim, 2006:35) 6 Referente al pop coreano que surgió desde Seo Taiji and Boys hasta la actualidad 
"Las afinidades culturales regionales también ayudan a explicar este fenómeno en el sentido que el éxito de la Ola coreana está estrechamente relacionado con la capacidad de la cultura y los medios coreanos de traducir la cultura americana u occidental para encajar en el gusto asiático" (p.145).

Por lo tanto, la capacidad de innovar a partir de lo "conocido" entendiendo este como lo occidental y la capacidad de negociación con los patrones establecidos para ser adaptados, construyen identidad nacional.

\section{Difusión en Asia del Este}

omo se mencionó anteriormente, la liberalización de los medios en Asia actuó como catalizador de la circulación trasnacional y el consumo de la cultura popular coreana a nivel inter-asiático. La difusión de los productos culturales coreanos se daría de manera rápida y progresiva teniendo en cuenta que, debido a la proximidad geográfica con países como Japón, Taiwán, Hong Kong, Vietnam, Tailandia, Indonesia, Filipinas, Singapur y otros países del sudeste asiático, comparten elementos culturales distintivos como los valores confucianos, religión, procesos históricos y de guerra en países, por mencionar algunos. Incluso, de manera clandestina al régimen de Corea del Norte, siendo consumida por las nuevas generaciones

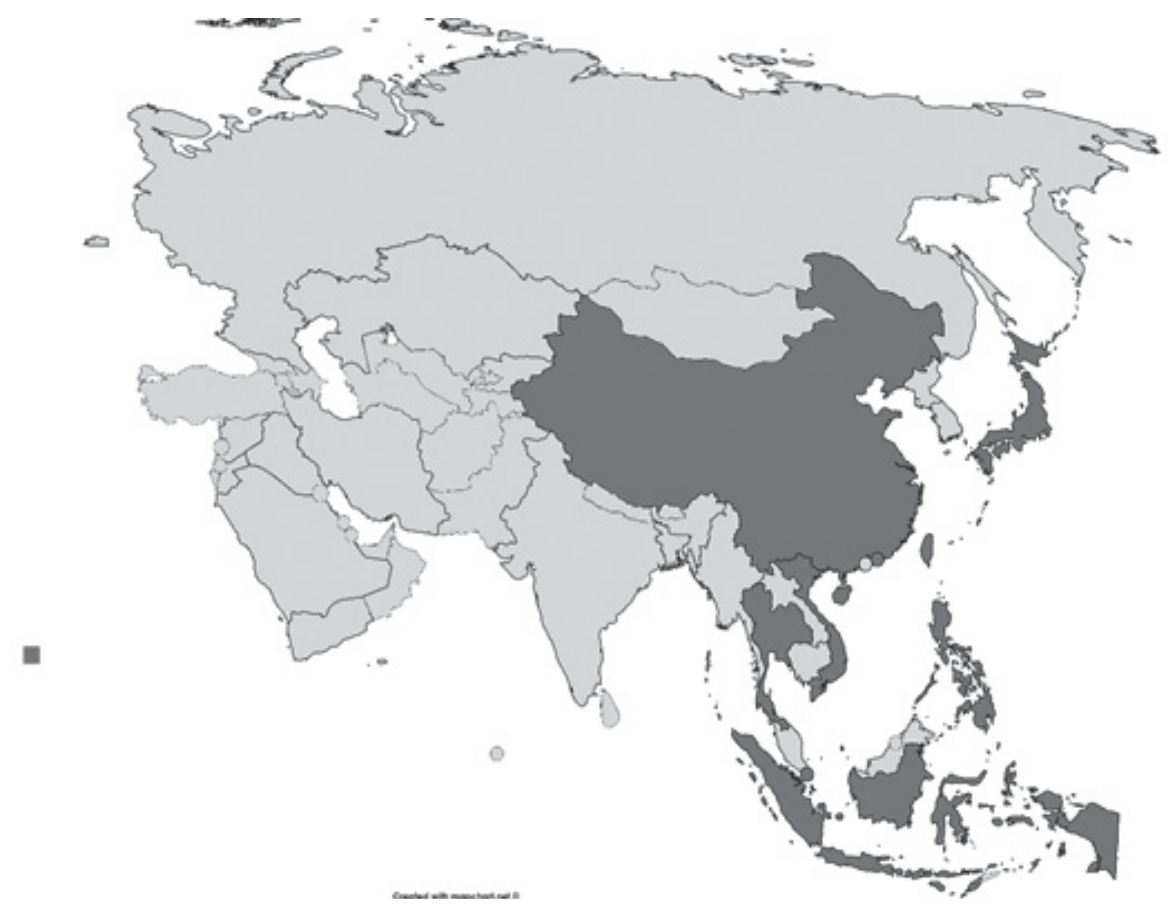

Mapa 2: Difusión en el sudeste asiático

Fuente: Elaboración propia de clase alta. (Kim, 2011: 130), que en palabras de López (2012) posibilitaría el posicionamiento de la cultura popular coreana como "referente en el código cultural de Asia en la actualidad".

Para entender el contexto en el que Corea se relaciona con sus vecinos y cómo afecta que este haya ocupado un lugar predominante en el mercado cultural asiático, es menester indicar que la relación con China y Japón es catalogada 
como "de amor y odio" (Lee, 2011:58) teniendo en cuenta su particular vínculo histórico. La hegemonía china, el colonialismo japonés en Corea (1910-1945) y en Asia, la guerra de Corea (1950-1953) y la división de la península son, por mencionar, algunos hechos que han marcado las relaciones de estos países, los cuales fueron hostiles o indiferentes a todo tipo de manifestación cultural. Tal fue el grado de hostilidad entre Corea y Japón que la importación de productos de la cultura popular japonesa estuvo prohibida por el gobierno de Corea hasta 1998 debido al pasado colonial entre estos países. (Kim, 2007: 124).

Como consecuencia de la inmensa popularidad alcanzada por la cultura popular coreana en tan corto tiempo logrando competir y "desplazar" a las culturas dominantes, Lee (2011) sugiere, a partir de su investigación sobre el cubrimiento mediático de la Ola coreana, un posible "imperialismo cultural" coreano en un mercado donde Japón y su industria cultural consolidada era dominante. En la prensa coreana se evidenciarían esfuerzos por mejorar la imagen nacional, siendo Hallyu el principal elemento para la creación de la imagen de marca nacional, mostrado con orgullo nacional, honor y triunfo. Sin embargo, ¿Qué tan factible es que Corea pueda reemplazar la hegemonía cultural de Japón en Asia, una cultura popular consolidada y con una amplia variedad de productos de consumo incluso a nivel transnacional? ¿Es posible que dos culturas convivan sin excluirse la una de la otra? ¿Qué tan adecuado es el sentimiento de Japón para temer a la "coreanización" de su cultura popular? El rechazo en etapas primarias de la consolidación de Hallyu por parte de Japón no es más que un posible efecto negativo propio del proceso de hacer más abierta y global a la cultura popular coreana.

En este país, el primer drama o telenovela en llegar a las pantallas fue Winter Sonata o Sonata de inverno en 2003, emitido por la cadena televisiva Japanese Broadcast Corporation (NHK) en el horario habitual de los productos importados de Estados Unidos. Este, representaría un puente entre Corea y Japón que los políticos de turno no hubieran sido capaces de establecer. Por su parte, en China a principios de los 90 surgiría una gran demanda de programación debido al rápido crecimiento de las industrias mediáticas, posibilitando la creación de nuevos canales. A su vez, fomentaría un ambiente propicio para que las producciones coreanas acapararan el mercado; que, por su contenido y aceptación, se reafirman hoy en día en las pantallas de ese país. Sin embargo, la experiencia en Hong Kong es diferente, puesto que ver un drama coreano tendría la connotación de ser más "terrestre", aquello debido
7 Variación del término inglés "Americanization", que empleo para hacer referencia a la influencia de la cultura popular coreana sobre otras culturas asiáticas. 
8 También conocido como horario triple A (AAA). Se utiliza para hacer referencia al horario donde se registra una mayor audiencia. a la adquisición de programas que las compañías nacionales transmitirían en el horario primetime ${ }^{8}$. (Kim, 2007:126-129)

No obstante, el medio de consumo más popular para la época en estos tres últimos países serían los Video Compact Disc (VCD), muchos de los cuales eran distribuidos de forma pirata y con subtítulos en chino para el caso de la audiencia de Taiwán. Debido a su naturaleza portable, sería ampliamente acogida por estudiantes y trabajadores de tiempo completo. (Kim, 2011:128129) En este sentido, se puede catalogar los VCD como un canal de distribución alternativo a los medios dominantes que, competiendo por captar el mayor número de audiencia, en materia económica produjo el considerable aumento en los precios de dramas y, por ende, mayor grado de circulación transnacional y consumo de los mismos. Esta alternativa, que más tarde sería sustituida por el internet y los portales especializados en streaming con subtítulos, permite la autonomía de consumo por parte del espectador.

\section{Difusión cultural y soft power}

T a penetración de Hallyu en el este y sudeste asiático ha tenido consecuencias positivas para las relaciones internacionales de Corea, en la que la cultura es el principal motor y funciona como instrumento mediador en asuntos de política exterior. Para ese país, ha sido un pretexto para el fortalecimiento del nacionalismo históricamente desquebrajado a causa del colonialismo japonés, la guerra y las tensiones propias de la división de la península. De hecho, Doboo (2006) describe que las estrellas del pop han ayudado a mejorar las relaciones exteriores coreanas al mismo nivel que importantes personajes de la política coreana.

El origen del mencionado nacionalismo coreano puede situarse en tres etapas: la nación republicana (1919-1945), la nación revolucionaria en Corea del Norte (1950-2012) y la nación del desarrollo en Corea del Sur (1950-2012), teniendo en cuenta que la aproximación al carácter estatal y de nación como un sistema no se desarrolló en el caso coreano debido a dichos procesos. (Girouar, 2015: 70-73 citado en Kim \& Marinescu 2015: 7) En este contexto, el fenómeno Hallyu ha sido parte de ese sentimiento nacionalista propio de finales del siglo XX y comienzos de los 2000, cuando Corea se consolida como economía emergente en Asia después de la crisis al ser auxiliada por el Fondo Monetario Internacional (FMI), ingresa a la Organización para la Cooperación y el Desarrollo Económicos (OCDE) en 1996. Luego de su recuperación económica, el país creció rápidamente, y como miembro de la 
OCDE pasó a ser catalogado por el Banco Mundial como economía de altos ingresos principalmente por su economía industrializada ${ }^{9}$, y está clasificado por FMI como economía de alto ingreso, además de permitirle ser miembro del G-20 $0^{10}$. Adicionalmente, debido al impulso comercial de la cultura popular, Hallyu logró ser gestor al integrar cultura y economía para posicionar a Corea en la esfera pública internacional en beneficio de su desarrollo progresivo.

No obstante, la forma sutil en la Corea del Sur que ejerce poder sobre sus países cercanos para obtener un beneficio, que para el caso de Hallyu, ha sido una capacidad de atracción que Corea ha sabido emplear en el fomento de su cultura popular por medio de la admiración de sus valores nacionales. Joseph S. Nye (2003) entiende el poder desde tres dimensiones: el poder militar y el económico como poder duro o hard power y la forma indirecta de ejercerlo, desde el poder blando o soft power. Si bien el autor hace referencia a Estados Unidos cuando describe el concepto y señala que este es el modelo dominante en la exportación de cultura popular más que cualquier otro país, puede utilizarse en el caso coreano puesto que cumple con la principal premisa: "Si yo consigo que tú quieras hacer lo que yo quiero, entonces no tengo que obligarte a hacer lo que tú no quieres hacer (...) el soft power es la capacidad de atraer y actuar; y la atracción a menudo lleva a la conformidad o la imitación" (30-31).

Por lo tanto, no es simplemente una forma común de influencia y al mismo tiempo es más que un poder cultural. Es en palabras del autor un "resorte de poder", que de la mano de los valores consigue mediar en la expresión de la cultura, las políticas interiores y las actuaciones en el ámbito internacional. Otra implicación de este término es el señalado por Kim \& Marinescu (2015) que en los estudios culturales y sociales se utiliza como sinónimo de 'influencia cultural', que desde esta perspectiva se destaca la exportación de la cultura popular como el principal vehículo mediante el cual el mundo comienza a saber y es atraído por un país.

En la era de la hiper-conectividad, el soft power subyace como un componente esencial para la cultura con fines diplomáticos. Sin embargo, ¿qué características debe tener una corriente cultural para aumentar su soft power? ¿Cuál es el "secreto del éxito"? Primeramente, debe tener su cultura e ideas dominantes más cercanas a la normal establecida por la predominancia global (el liberalismo, el pluralismo, y la autonomía); seguidamente debe poseer canales efectivos de acceso a la información; y por último, debe ser capaz de
9 Datos recuperados de http://datos.bancomundial.org/pais/ corea-republica-de 10 Foro de discusión, planificación y supervisión de la cooperación económica internacional constituido por los países más industrializados (8), los recientemente industrializados (11) más la Unión Europea (UE). 
11 En México, debido a las políticas y leyes migratorias de los períodos presidenciales de Porfirio Díaz orientadas a legalizar, registrar y normalizar el ingreso de extranjeros (Romero, 2000: 150).

12 La autora Corina Courtis (2010) utiliza este término para describir la discriminación propia de las interacciones cotidianas de la colectividad coreana, causada por su fenotipo en el contexto netamente argentino. Se considera pertinente para este análisis rescatar este concepto para una comprensión más amplia de la presencia coreana en Latinoamérica, propiamente en Argentina, México y Perú. transmitir credibilidad a sus pares en asuntos domésticos e internacionales (Nye, 2003: 105). Por lo tanto, Corea cumple con las exigencias del mercado cultural global a pesar de no sentirse eclipsada por su hermana del Norte que acapara las miradas de la prensa internacional debido a sus prácticas militares y nucleares. Corea es entonces prueba fehaciente que desde la cultura popular se puede cambiar la imagen de un país, manteniéndola vigente y en constante cambio a partir de procesos de asimilación e influencia propios de una cultura global.

\section{Hallyu en Latinoamérica: relaciones bilaterales, comerciales y migraciones}

n América Latina el proceso de penetración de Hallyu ha sido particularmente diferente al asiático. A simple vista las barreras idiomáticas, culturales y geográficas serían elementos indiscutibles para un posible rechazo. Por lo tanto, el proceso para los distintos países ha estado mediado por tres factores que interrelacionados han condicionado la aceptación de los productos audiovisuales propios de la cultura popular coreana: las relaciones diplomáticas, las relaciones comerciales y los procesos migratorios.

Si bien México y Argentina son históricamente los países latinoamericanos con mayor índice de inmigración coreana en los siglos XIX y XX ${ }^{11}$, países como Chile, Venezuela, Panamá, El Salvador, Ecuador, Costa Rica, Paraguay, por mencionar algunos, han acogido las telenovelas provenientes de Corea. No obstante, es importante mencionar la fuerte presencia asiática en Perú, lo que lo añade al grupo de Argentina y México como los tres países latinoamericanos con mayor afinidad hacia los productos de la cultura popular coreana. Es interesante encontrar que en estos tres países el común denominador en la inmigración coreana se presenta desde dos tipos de colectividades: aquellos coreanos de nacimiento, y los "coreanos étnicos"12 los hijos de inmigrantes nacidos en territorio latinoamericano (Courtis, 2010) que serán portadores de la cultura de sus padres en nuevo ambiente, el del país donde nacieron.

En ese sentido cabe resaltar que la diáspora coreana a América Latina y la presencia coreana ha sido poca si se compara con los Estados Unidos, Australia, y otros países asiáticos, teniendo en cuenta la coyuntura de la Guerra Fría y la inestabilidad política de la península. En México, la migración coreana data de 1905 con la intención de lograr rápidamente poder adquisitivo y regresar a ese país para adquirir prestigio, mejores condiciones educativas 
y sentimiento nacionalista (Romero, 2000:151) y supliendo la necesidad de mano de obra del país azteca. Es claro que los conflictos políticos internos y avance imperialista por parte Japón, serían hechos que también tendrían cierta repercusión en la decisión de los coreanos de dejar o no su país.

No obstante, Romero (2000) resalta que, en comparación con la llegada de las comunidades chinas y japonesas a México, la coreana fue poco significativa debido al reducido número de inmigrantes, ante la imposibilidad de retornar a su país, terminaron por adaptarse a la sociedad mexicana venciendo las barreras culturales a través de matrimonios mixtos.

En Argentina, el asunto de las migraciones funcionó a base de redes. Estas se remontan hasta la segunda mitad del siglo XX cuando a principios de los años 60 comenzaron las primeras inmigraciones de carácter agrario que fueron planeadas desde Corea, destinados en un principio a otros países de Sudamérica, entre ellos Brasil y Paraguay, que no prosperaron y terminaron por asentarse en ese país. Diez años más tarde, se produciría una masiva llegada. Esta vez bajo la firma del Acta de Procedimiento para el ingreso de inmigrantes coreanos a la Argentina, pacto de ambos gobiernos y con aportes de capitales de los mismos. En 1990, la tercera llegada sería de forma no sistematizada respondiendo a redes religiosas, y en la actualidad debido a las redes de globalización (Trincheri, 2010).

En Brasil la primera oleada de inmigrantes coreanos llegaría como parte de ese proceso planificado en 1962 con el apoyo del Gobierno coreano, y se extendería hasta 1966. Es así como se asientan primeramente en zonas agrícolas para después moverse hacia las urbes, como São Paulo, debido a la poca experiencia en este sector, dedicándose principalmente a la industria textil. Para la década del 70, se permitiría la entrada de coreanos únicamente portadores de título universitario, técnico o con contrato de trabajo. En contraste, el proceso en Perú se daría a partir de la re-emigración de países como Bolivia, Chile, Argentina o Paraguay (Mera, 2005).

A simple vista, las relaciones de Corea comenzarían a establecerse de forma sistemática en los años 60 con América Latina. En 1959 con Brasil ${ }^{13}$, en 1962 con México y Chile, un año después con Argentina ${ }^{14}$ y Perú, pero con intereses específicos: establecer convenios con países latinoamericanos con el fin de favorecer los procesos migratorios de sus ciudadanos. Hoy en día las relaciones bilaterales se producen con objetivos diferentes. Quizá el país
13 Fue el primer país latinoamericano en proceder al reconocimiento oficial de $\mathrm{Co}$ rea del Sur, y en tener embajada en las dos Coreas.

14 Cabe resaltar que según el Decreto 1709 de 1977, la República de Argentina rompe relaciones con la República Popular Democrática de Corea (Corea del Norte), previamente establecidas en 1973. 


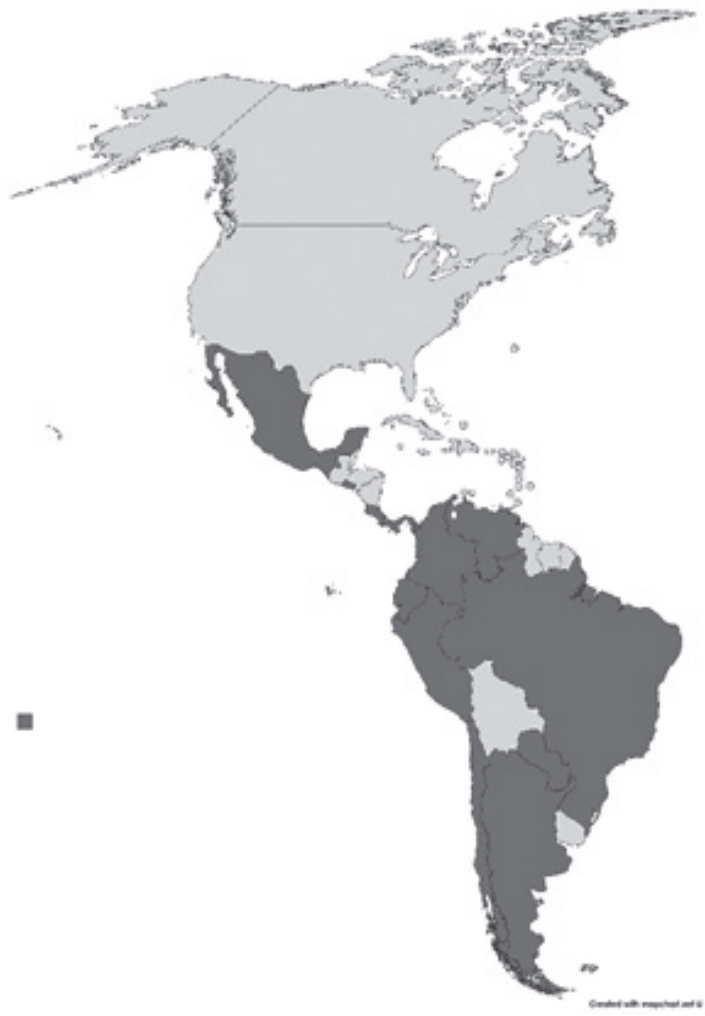

Mapa 3: Difusión en América Latina

Fuente: Elaboración propia

latinoamericano con el que tenga mejores relaciones es México ${ }^{15}$, que por su tamaño similar y el tipo de industria que los caracteriza pueden entenderse como economías similares competitivas. Corea propende por establecer tratados de libre comercio (TLC), o en su defecto acuerdos (ALC) como en el caso del Perú vigente desde 2011. Con Chile, por ejemplo, el tratado está vigente desde 2004, y en el contempla un acuerdo de intercambio de producciones entre la Televisión Nacional de Chile (TVN) y el Sistema de Radiodifusión de Corea (KBS), lo cual favorece aún más la llegada de producciones coreanas a las pantallas chilenas sin coste representativo.

Sin embargo, hasta la fecha está en negociación con México, y con Colombia el acuerdo se firmó oficialmente en 2013 y solo hasta 2017 entró en vigor. En este acuerdo, se destaca que en materia de cooperación cultural (art 17.13) se acuerdan considerar y negociar acuerdos de coproducción de programas audiovisuales entre ambos países. Con Colombia, sin embargo, el caso es particular puesto

15 En 2001 se estableció la Comisión México- Corea Siglo XX para estudiar las perspectivas de la relación bilateral y presentar estrategias y acciones a mediano a largo plazo. En 2006, se constituyó la Alianza Estratégica para la Prosperidad Mutua entre ambos países, con el fin de coordinar acciones en foros multilaterales con mayor frecuencia. Sus relaciones culturales se basan en un convenio cultural firmado en 1966. (Embajada de México en Corea, n.d) Disponible en: https://embamex sre.gob. $\mathrm{mx} /$ corea/index.php/la-embajada/ 2016-04-27-03-35-47 16 Disponible en http://nacion.com/ economia/politicaeconomica/Costa-RicalanzamientoCoreaSur 01484251585 html Recuperado el 13 de marzo de 2016. que estableció formalmente relaciones en 1962 después de haber sido el único país latinoamericano en enviar tropas a luchar en la Guerra de Corea por el ejército de la ONU.

A partir de lo anterior, podría afirmarse que México, Perú, Argentina y Brasil son países claves para la llegada de Hallyu en Latinoamérica debido a la estrecha relación histórica con la sociedad coreana tras haber sido destino de inmigración de sus nacionales y al vínculo considerable en materia económica y diplomática, estuvieron más propensos a la aceptación de muestras culturales y por tanto de la Ola coreana, hecho que extraña al conocer que el primer drama o telenovela coreana fue transmitido en Costa Rica en 2001, país con el que Corea también estableció relaciones oficiales en 1962 pero que no equipara en materia de migraciones y acuerdos. Solo hasta el primer semestre de 2015 iniciaría negociaciones para un posible acuerdo de asociación con este país ${ }^{16}$.

El siguiente destino de Hallyu en Latinoamérica sería México. Se considera inicialmente que el fenómeno comenzó con la difusión y la promoción inicial 
de las telenovelas o dramas coreanos. López (2012) asegura que es posible indicar la llegada de estos productos asiáticos para el 2002 con la primera transmisión de "Una estrella en mi corazón" en la televisión mexicana a través del canal 34 o Televisión Mexiquense, perteneciente al gobierno del Estado de México que emitió su señal a un público relativamente amplio; al igual que con "Todo sobre Eva" ese mismo año. Seguidamente, con la transmisión en 2005 de producciones como "Sonata de invierno" y "Escalera al cielo" ampliamente conocidas por su gran acogida en el público asiático, y en 2006, "La joya en el palacio", generaron un hito que permitiría al público mexicano enriquecerse de la cultura de este país a partir de las historias presentadas a través de las pantallas, aunque no se tienen datos de la franja en la que fueron emitidas.

A partir de la aceptación de este tipo de producciones, se abrió paso para la llegada de otros productos de entretenimiento a México en su orden de difusión, principalmente: películas, en su mayoría desde la categoría de cine arte o en festivales de cine; y la música, que a pesar de la barrera del lenguaje se ha popularizado a la par de las telenovelas o dramas. López (2012) apunta que es quizá el elemento de más fácil y rápida dispersión mediante los recursos virtuales disponibles en la actualidad, y de más rápida asimilación debido a la búsqueda de nuevos dramas coreanos en internet. Para esta autora, otros productos han tenido menor impacto, pero no por ello menor importancia. En este sentido, Corea se jugaría la "carta de aceptación" de su cultura popular en los mercados latinoamericanos con las telenovelas o dramas.

Sin embargo, afirmar que la llegada del Hallyu a México, como a otros países de Latinoamérica, se dio a partir de la transmisión en televisión nacional sería una forma de coartar la difusión por otro tipo de canales, tal como sucedió en el caso de Hong Kong y el taiwanés mencionado anteriormente en el que los canales alternativos de distribución sirvieron como plataforma para impulsar y consolidar el éxito de los dramas o telenovelas. En ese sentido, se podría catalogar como la llegada oficial de este fenómeno a las pantallas, si no se tiene registro alguno de la disponibilidad web o consumo de estas antes de la fecha indicada por la autora.

En el resto de Latinoamérica, la llegada de las telenovelas o dramas se dio de manera sincronizada en algunos países. Iadevito y Bavoleo (2015) recopilan de manera acertada esta información. Primeramente, es posible destacar que 
17 Disponible en http://exitoina. perfil.com/201603-06-390151-el-verano-mas-economicode-lahistoria-televisiva Recuperado el 7 de marzo de 2016

18 Disponible en http://www.clarin. com/extrashow/tv/ Telenovelas-coreanas-boom-vinolejos $0 \quad 1536446778$. html Recuperado el 7 de marzo de 2016
México ha sido el país con mayor número de transmisiones de las telenovelas o dramas coreanos en Latinoamérica con un total de diez -incluida la retransmisión de "Una joya en el palacio" en 2009, y Costa Rica le sigue los pasos con nueve. Por su parte, Perú acumula unas siete trasmisiones y Paraguay seis. Entre los lugares más bajos en la lista de la programación se sitúan Chile y Panamá con cinco, Ecuador con cuatro, y Venezuela con tan solo tres.

De acuerdo a sus gráficos elaborados por estas autoras, puede establecerse que la primera "oleada" se dio efectivamente entre el 2002 y 2003 en México, Perú, Chile y Panamá. La segunda "oleada" podría catalogarse de 2005 al 2008, esta vez incluyendo la transmisión en países como Venezuela, Ecuador y Paraguay. Consecuentemente, entre el 2010 y el 2013 se presentaría la tercera en todos los países mencionados anteriormente. Es importante destacar el año 2012, sería el mejor para la cultura popular coreana en Latinoamérica, puesto que, debido al gran éxito de sus producciones, se transmitirían dos en Panamá, dos en Paraguay, una en Ecuador, dos en Chile, y dos en Costa Rica. Para el 2013 se transmitiría una producción en Paraguay y una en Venezuela. Y, México finalmente sería el único país en transmitir una producción coreana para el 2015. Es menester resaltar una característica esencial de estas transmisiones es que se hacen dobladas al español y no en su idioma original subtitulado.

Es común por lo visto, que tras el éxito en audiencia las cadenas televisivas latinoamericanas decidan retransmitir una telenovela o drama coreano, tal como ha sucedido con producciones como Escalera al Cielo en varios países, re-emitida en 2016 Argentina por Telefe, canal de televisión abierta, en su franja de la tarde. Sin embargo, se debe tener en cuenta el factor económico en este tipo de programaciones, que devela el bajo presupuesto invertido tras el bajísimo costo de su adquisición a causa de un acuerdo con Telemundo, permitiéndole comprar tres telenovelas o dramas coreanos, siendo My Love From Another Star o "Mi amor de las estrellas" (2013) transmitida en el primer semestre de 2016 en la misma franja que Escalera al cielo, y al ser la segunda producción coreana en emitirse por este canal. Es también de anotar que en 2015 la Embajada de Corea en este país cedería sin cargo Jardín secreto a la señal de cable Magazine ${ }^{17}$. En ese mismo año, se transmitió Angel Eyes o "Mirada de ángel" (2014) después de que "Mi amor de las estrellas" fuera seleccionada por el público en un sondeo desde la cuenta de Telefe en Twitter para salir al aire primero ${ }^{18}$ (Bruno, 2016). 


\section{Caso Colombia}

F

n el contexto latinoamericano, el 2012 se consolida como un año estratégico para la cultura popular coreana, siendo el año donde más

transmisiones se registraron en todo el continente. Si bien es cierto que los dramas o telenovelas coreanas se emitieron por primera vez en la primera década de los 2000, en Colombia la llegada de forma masiva se presenta de forma tardía puesto que la primera transmisión de este tipo de producciones se registra a partir de 2012 en donde se sitúa a RCN Televisión, canal de carácter privado que se emite por televisión abierta, como la cadena por excelencia que las ha transmitido hasta la fecha.

En contraste, se podría catalogar que la primera llegada a la televisión colombiana se dio con All about Eve o "Todo sobre Eva" (2000) emitida en el 2002 por el canal regional Canal Capital, y posteriormente en el 2010 transmitirían "Una joya en el palacio" (2003) aunque la audiencia alcanzada se limitaría a Bogotá y su área metropolitana, zona geográfica donde se recibe su señal por ser de carácter público. Además, se tienen registros de que una cadena regional transmitió en 2007 la popular "Escalera al cielo"19, posiblemente sea Canal Capital debido a las producciones coreanas emitidas mencionadas. Sin embargo, el verdadero éxito de esta última se daría con la transmisión por RCN en octubre de 2012 en la franja vespertina, horario históricamente ocupado por las telenovelas mexicanas y siendo el primer drama emitido por ese canal a partir de una apuesta por atraer más audiencia.

Se tiene certeza de que RCN ha transmitido en total siete dramas o telenovelas coreanas que se relacionan a continuación en el orden cronológico de su emisión: En 2012, después del éxito con "Escalera al cielo" (2002) -que de hecho superó a "Tu voz estéreo" programa de producción nacional de competencia en Caracol Televisión y se posicionó como una de las producciones más vistas- se transmitiría en noviembre $A$ Thousand Day's Promise o "Mil días de promesas" (2011). Para 2013, RCN transmitiría cuatro telenovelas o dramas coreanos: My Man's Woman o "La mujer de mi esposo" (2007) en enero, King of Baking o "Pan, amor y sueños" (2010) en marzo, Secret Garden o "El jardín secreto" (2010) transmitida en mayo, y por último Spring Waltz o El vals de primavera (2006) en agosto. Y finalmente en 2014 se emitiría Autumn in my Heart o "Otoño en mi corazón" (2002) en la franja de la tarde. Pese a los altos índices de audiencia, se desconocen las razones por las cuales RCN Televisión dejó de transmitirlas. Para los años siguientes retornarían a su lugar las telenovelas mexicanas, y en cambio comenzaría a transmitir

19 Recuperado el 30 de marzo de 2016 Disponible en http:// es.doblaje.wikia.com/ wiki/Escalera_al_cielo 
telenovelas brasileñas, teniendo gran éxito con "Avenida Brasil" en 2014 en el horario prime time.

No obstante, el artículo 4 de la ley 680 de 2001 que modifica al artículo 33 de la ley 182 de $1995^{20}$ establece que: "cada operador de televisión abierta y concesionario de espacios en los canales de cubrimiento nacional, deberá cumplir trimestralmente los siguientes porcentajes mínimos de programación de producción nacional: a) Canales nacionales: De las 19:00 horas a las 22:30 horas (triple A), el 70\% de la programación será producción nacional. De las 22:30 horas a las 24:00 horas, el 50\% de la programación será de producción nacional. De las 00:00 horas a las 10:00 horas, el 100\% de la programación será libre. De las 10:00 horas a las 19:00 horas el 50\% será programación de producción nacional". Esto en función de proteger la prevalencia de la producción colombiana en las pantallas ante la presencia de producciones extranjeras en las programaciones de los canales nacionales.

Por su parte, Caracol Televisión, canal privado en Colombia con emisión abierta que se disputa la audiencia con RCN Televisión, firmaría en 2011 un acuerdo de cooperación con Arirang TV, televisora coreana operada por la Korea International Broadcasting Foundation, donde buscarían intercambio de contenidos en el área cultural y de noticias. De este acuerdo sería fruto un año más tarde, justamente el mismo año de la primera emisión de una telenovela o drama coreano en la televisión abierta colombiana, la producción de un programa-concurso o reality show dedicado al K-pop que elegiría a un grupo de seis finalistas para viajar a Corea para conocer los grupos de este género también patrocinado por LG Electronics (Koreanet, 2012). Y es de resaltar que, en materia de producciones internacionales, Caracol ha optado por añadir a su parrilla de programación a las telenovelas turcas también en la franja de la tarde desde el segundo semestre de 2015. Es para destacar, sin embargo, que después de tres años de brecha, Caracol, transmitió en enero de 2018 Angels Eyes en el horario de media noche, hecho que pudo haber estado influenciado por el vigor del TLC entre los dos países.

20 Ley que crea la televisión privada en $\mathrm{Co}-$ lombia.

\section{Conclusiones}

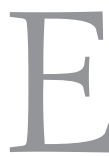

n América Latina el proceso de penetración de Hallyu ha sido mediado por las relaciones diplomáticas, las relaciones comerciales y los procesos migratorios (diáspora coreana). No es complemente determinante pero sí tiene alta incidencia que México, Argentina, Perú y Brasil, países que históricamente han acogido población asiática y con los cuales las relaciones 
bilaterales (comerciales y diplomáticas) son fuertes y estables, tengan registrado un mayor número de dramas coreanos transmitidos en televisión abierta, y por lo tanto fuesen más propensos a la aceptación de la Ola coreana.

En Colombia, por lo tanto, se tiene la hipótesis de que estos factores no determinaron la llegada, puesto que esta se dio de manera tardía si se compara con sus países vecinos. En el 2012, año estratégico para la cultura popular coreana por el número de transmisiones que se registraron en todo el continente, llega de forma masiva al país. Se tiene certeza que RCN ha transmitido en total siete dramas o telenovelas entre 2012 y 2014 en el horario tradicionalmente ocupado por las telenovelas mexicanas.

De esta forma, se demuestra la presencia y penetración de Hallyu en Colombia a partir de la transmisión de productos audiovisuales propios de la cultura coreana en dos de los canales privados más importantes y con mayor audiencia en el país, que, aunque con diferentes contenidos marcarían una pauta como primer acercamiento de la televisión colombiana a Corea.

\section{REFERENCIAS BIBLIOGRÁFICAS}

ÁLVAREZ, M. (2011) "Dorama japonés como material auténtico para la enseñanza: Un análisis comparativo de las telenovelas de Japón y Colombia” en Memorias del XIII Congreso Internacional de ALADAA, Asociación Latinoamericana de Estudios de Asia y África, Disponible en: http://ceaa. colmex.mx/aladaa/memoria_xiii_congreso_internacional/images/alvarez_mo nica.pdf

BUSTElO, P. (1991) La expansión de las grandes empresas de Corea del Sur («Chaebol»): un ejemplo de estrategia corporativa. Cuadernos de Estudios Empresariales (1) p. 1323.

COURTIS, C. (2010) Migración y discriminación en la Argentina: Un diagnóstico participativo. Cuadernos Judáicos (27) p.1-10

IADEVITO, P., \& Bavoleo, B., (2015) Telenovelas coreanas en América Latina una aproximación desde los estudios culturales. Revista Digital Mundo Asia Pacífico. 4 (6) DOI: http://dx.doi. org/10.17230/map.v4.i6.02 Koreanet (2012) Apasionados seguidores del K-pop en todo el mundo. En SpanishKorea.net del 31 de Mayo de 2012. Recuperado el 29 de Marzo de 2016. Disponible en http://spanish.korea.net/NewsFocus/Culture/view?articleId=100605

KIM, Y. (2007) "The rising of East Asia 'Wave': Korean media go global" en Daya Thussu (ed) Media on the Move: Global Flow and Contra-Flow. (pp. 121-135) Londres y Nueva York: Routledge.

KIM, Y. \& Marinescu, V. (2015) Mapping South Korea's Soft Power: Sources, Actors, Tools and Impacts. Romanian Journal of Sociological Studies. 1:3-12. 
LEE, S. (2011) The Korean Wave: The Seoul of Asia. The Elon Journal of Undergraduate Research in Communications. 2(1):85-93.

LÓPEZ R, N. (2012) "Hallyu y su impacto en la sociedad mexicana" Estudios Hispánicos 64:579_ 598.

MERA, C. (2005) "Diáspora coreana en América Latina", II Encuentro de Estudios Coreanos en América Latina, México, Colegio de México - Korea Foundation http://www.uba.ar/ceca/download/mera.pdf

NYE, J. S. (2003) La paradoja del poder norteamericano (G. Bustelo, Trad.). Madrid: Taurus

ROMERO, F. (2000) Factores que provocaron las migraciones de chinos, japoneses y coreanos hacia México: Siglos XIX y XX. Revista de Ciencias Sociales (Cr), vol. IV, núm. 90-91, pp. 141-153 Universidad de Costa Rica San José, Costa Rica. Disponible en:

http://www.redalyc.org/articulo.oa?id=15318269004

RYOO, W. (2009) Globalization, or the logic of cultural hybridization: the case of the Korean Wave. Asian Journal of Communication. 19 (2): 131-151, DOI: 10.1080/01292980902326427

SHIM, D. (2006) Hybridity and the rise of Korean popular culture in Asia. Media, Culture and Society. 28(1): 25-44.

TRINCHERI, A. (2010) "La inmigración coreana en la Argentina. Una migración de redes Asociación Latinoamericana de Estudios de Asia y África", en: XIII Congreso Internacional de ALADAA. Asociación Latinoamericana de Estudios de Asia y África, Disponible en: ceaa.colmex.mx/aladaa/ memoria_xiii_congreso/trincheri.pdf 